\title{
Cytotaxonomy and DNA taxonomy of lizards (Squamata, Sauria) from a tropical dry forest in the Chamela-Cuixmala Biosphere Reserve on the coast of Jalisco, Mexico
}

\author{
RICCARDO CASTIGLIA ${ }^{1,6}$, FLAVIA ANNESI ${ }^{2}$, ALEXANDRA M.R. BEZERRA $^{3}$, ANDRÉS GARCÍA ${ }^{4}$ \\ \& OSCAR FLORES-VILLELA ${ }^{5}$ \\ 'Dipartimento di Biologia Animale e dell'Uomo, Università di Roma “La Sapienza”, via A. Borelli 50, 00161, Rome, Italy. \\ E-mail: castiglia@uniromal.it \\ ${ }^{2}$ Dipartimento di Biologia Animale e dell'Uomo, Università di Roma “La Sapienza”, via A. Borelli 50, 00161, Rome, Italy. \\ E-mail: flavia.annesi@uniromal.it \\ ${ }^{3}$ Departamento de Zoologia, Universidade de Brasília, ICC Sul, CEP 70910-900, Brasília, DF, Brazil. E-mail: abezerra@fst.com.br \\ ${ }^{4}$ Estación de Biología Chamela Instituto de Biología, UNAM. Apdo. Postal 21. San Patricio, Jalisco, 48980 \\ ${ }^{5}$ Museo de Zoología, Facultad de Ciencias, UNAM A.P. 70-399, 04510.D.F., Mexico.E-mail: ofv@hp.fciencias.unam.mx \\ ${ }^{6}$ Corresponding author
}

\section{Table of contents}

Abstract

Resumen

Introduction

Material and methods

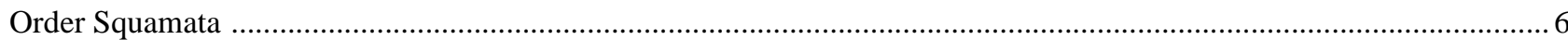

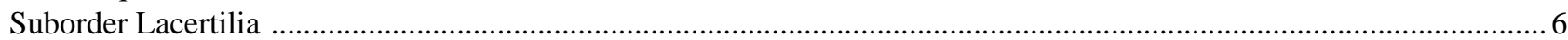

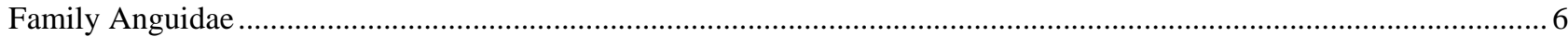

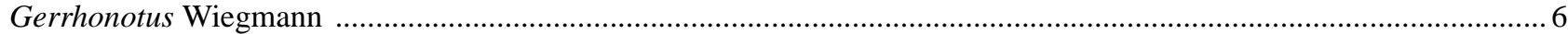

Gerrhonotus cf. liocephalus Wiegmann (Texas alligator lizard) .................................................................... 7

Family Eublepharidae

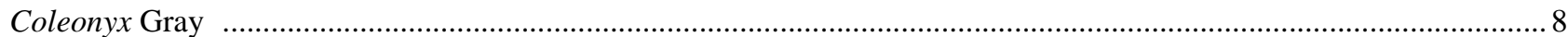

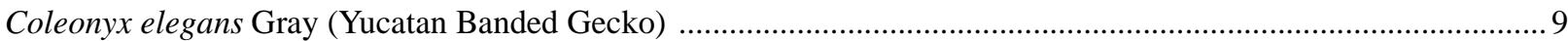

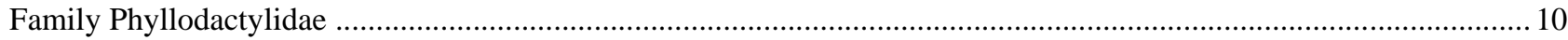

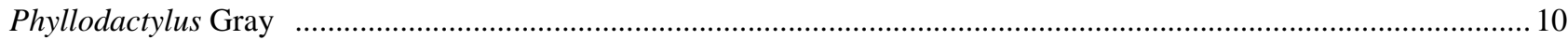

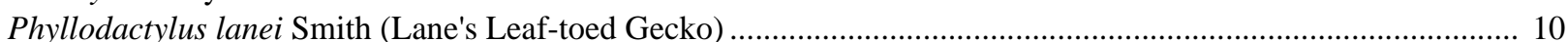

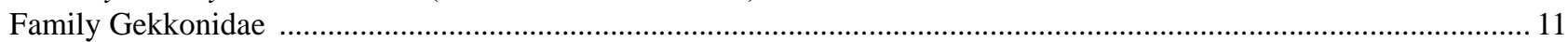

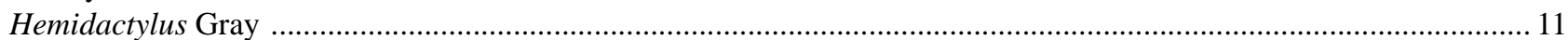

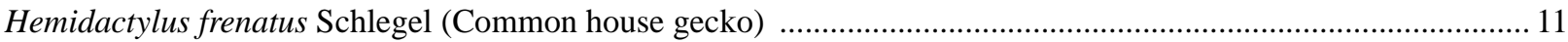

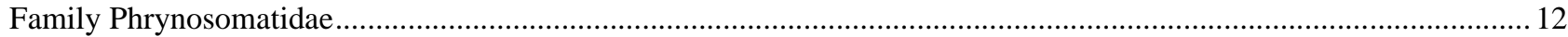

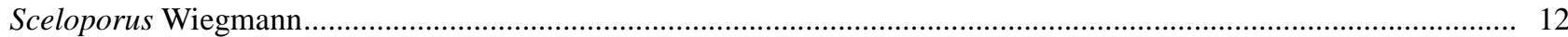

Sceloporus melanorhinus Bocourt (Pastel Tree Lizard) .................................................................................. 12

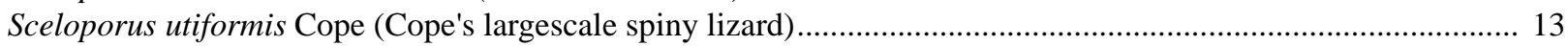

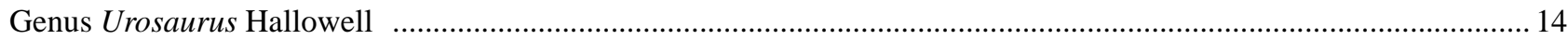

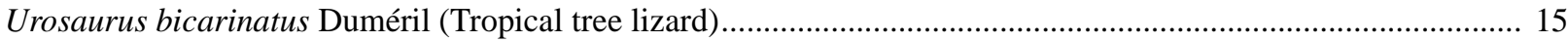

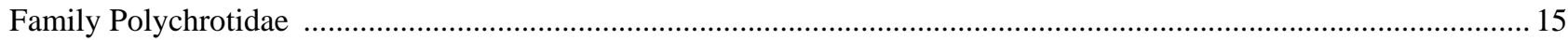

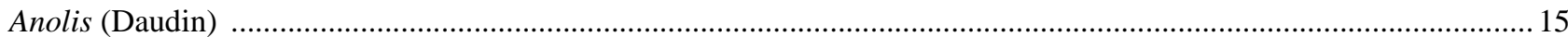

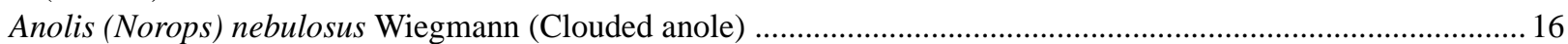

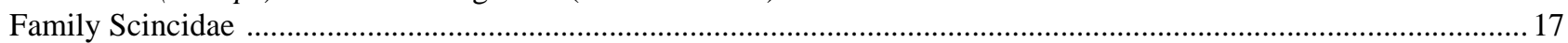

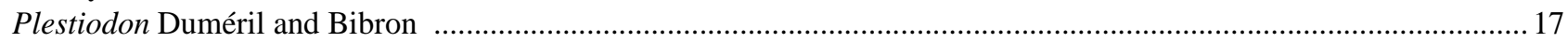

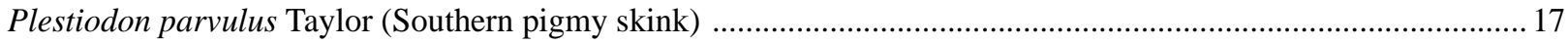

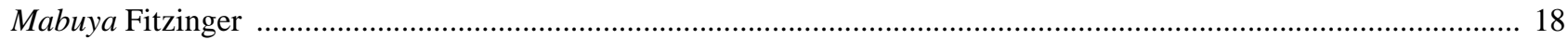

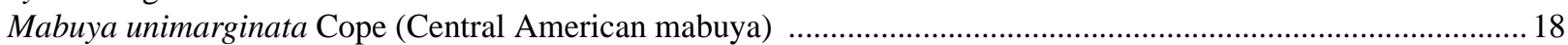


Family Teiidae 20

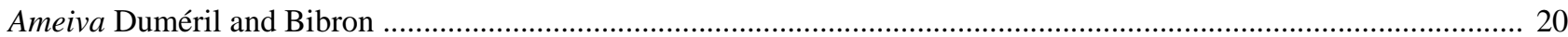

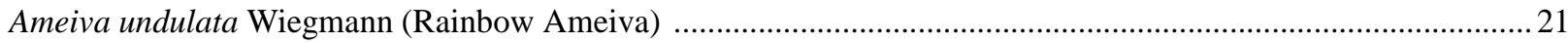

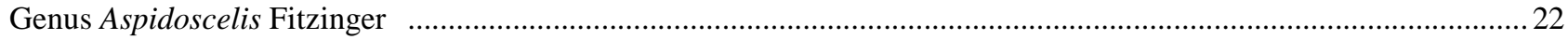

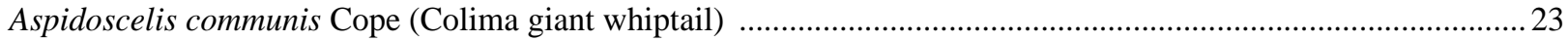

Aspidoscelis lineattissima (Cope) (Many-lined whiptail) ........................................................................23

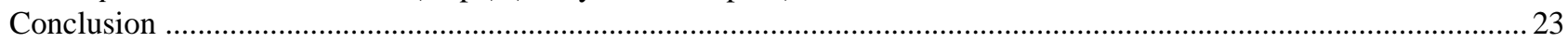

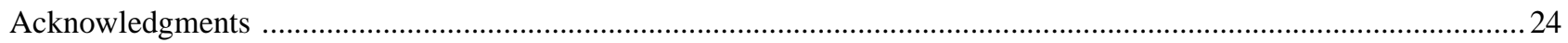

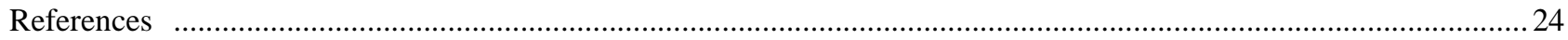

\begin{abstract}
Tropical dry forests contribute to a substantial proportion of the herpetological diversity of Mexico. The south-western coast of Jalisco is one of the more important areas by number of endemics and the high presence of endangered and restricted species. In this paper we used a combined karyological and molecular genetic (sequences of mtDNA genes for $\mathrm{NDH} 2$, cyt $b$ or $16 \mathrm{~S}$ rDNA) approach to genetically characterize 13 lizard species belonging to seven families that inhabit the dry forests of the Chamela-Cuixmala Biosphere Reserve (Anguidae: Gerrhonotus cf. liocephalus; Eublepharidae: Coleonyx elegans; Phyllodactylidae: Phyllodactylus lanei; Gekkonidae: Hemidactylus frenatus; Phrynosomatidae: Sceloporus melanorhinus, S. utiformis, Urosaurus bicarinatus; Polychrotidae: Norops nebulosus; Scincidae: Mabuya unimarginata, Plestiodon parvulus; Teiidae: Ameiva undulata, Aspidoscelis communis, A. lineattissima). The karyotypes of six species were here described for the first time (G. liocephalus, $2 \mathrm{n}=38,14$ macrochromosomes and 24 microcromosomes; $C$. elegans, $2 \mathrm{n}=24 \mathrm{FN}=26 ; N$. nebulosus $2 \mathrm{n}=30,13$ macro- and 17 microchromosomes; $M$. unimarginata $2 \mathrm{n}=32,18$ macro- and 14 microchromosomes; $P$. parvulus $2 \mathrm{n}=26,12$ macro- and 14 microchromosomes; A. undulata $2 \mathrm{n}=50,26$ macro- and 24 microchromosomes). Chromosomal heteromorphism was found in $C$. elegans, $N$. nebulosus, and S. melanorhinus. For P. lanei we found a karyotype different from that previously described in other localities. This variation matched with a high genetic divergence usually found in different species. The DNA typing of mtDNA genes allowed the identification of the taxonomic affinities of five Mexican endemic species, namely: $U$. bicarinatus, A. nebulosus, P. parvulus, A. lineattissima and A. communis. The specimen of Gerrhonotus from Chamela is very divergent by $16 \mathrm{~S}$ rDNA and probably does not belong to the so far studied species of Gerrhonotus. High genetic divergence has been also observed between samples of $A$. undulata and $U$. bicarinatus from different regions. In these latter two cases, additional data are needed to understand the taxonomic status of these populations.
\end{abstract}

Key words: biodiversity hotspot, cytogenetic, dry forest, molecular systematic, NDH2, Reptilia, 16S rDNA

\title{
Resumen
}

El bosque tropical caducifolio contribuye con una proporción considerable a la diversidad herpetológica de México. La costa suroeste de Jalisco es una de las áreas más importantes por su elevado número de especies endémicas y la alta incidencia de especies en riesgo y de distribución geográfica restringida. En este trabajo utilizamos un enfoque cariológico y genético molecular (secuencias de genes ADNmt para NDH2, cytb o 16S ADNr) para caracterizar genéticamente 13 especies de lagartijas pertenecientes a siete familias que habitan el bosque tropical caducifolio de la Reserva de la Biosfera Chamela-Cuixmala (Anguidae: Gerrhonotus cf. liocephalus; Eublepharidae: Coleonyx elegans; Phyllodactylidae: Phyllodactylus lanei; Gekkonidae: Hemidactylus frenatus; Phrynosomatidae: Sceloporus melanorhinus, S. utiformis, Urosaurus bicarinatus; Polychrotidae: Norops nebulosus; Scincidae: Mabuya unimarginata, Plestiodon parvulus; Teiidae: Ameiva undulata, Aspidoscelis communis, A. lineattissima). Aquí se describen por primera vez el cariotipo de seis especies ( $G$. liocephalus, $2 \mathrm{n}=38,14$ macrocromosomas y 24 microcromosomas; $C$. elegans, $2 \mathrm{n}=$ $24 \mathrm{FN}=26 ; N$. nebulosus $2 \mathrm{n}=30,13$ macro- y 17 microcromosomas; M. unimarginata $2 \mathrm{n}=32,18 \mathrm{macro}-\mathrm{y} 14$ microcromosomas; P. parvulus $2 \mathrm{n}=26,12$ macro- y 14 microcromosomas; A. undulata $2 \mathrm{n}=50,26$ macro- y 24 microcromosomas). Se encontró heteromorfismo cromosómico en C. elegans, $N$. nebulosus, y S. melanorhinus. En P. lanei encontramos un cariotipo distinto al descrito en otras localidades. Esta variación es similar a la que generalmente se encuentra entre especies con divergencia genética alta. La tipificación del ADN de los genes del ADNmt permitió la identificación de las afinidades taxonómicas de cinco especies endémicas de México, que son: U. bicarinatus, A. nebulosus, P. parvulus, A. lineattissima y A. communis. El espécimen de Gerrhonotus de Chamela es muy divergente por $16 \mathrm{~S}$ ADNr y probablemente no pertenece a las especies hasta ahora estudiadas de Gerrhonotus. También se observó alta divergencia genética entre las muestras de A. undulata y U. bicarinatus de diferentes regiones. Para estos dos últimos casos se requiere de datos adicionales para entender el estado taxonómico de estas poblaciones. 